\title{
Akupunktur ist etabliert
}

\section{Liebe Leserinnen und Leser, liebe Kolleginnen und Kollegen,}

ich freue mich sehr, Ihnen mit der 3. Ausgabe der Deutschen Zeitschrift für Akupunktur des Jahres 2019 das Produkt eines aktiven Redaktionsteams vorzulegen, das ich nach der Übergabe durch Tom Ots zum Halbjahreswechsel erstmals als Chefredakteur anleiten darf.

Bewährtes wird fortgeführt. Wir wollen im Rahmen von Schwerpunktthemen mit mehreren kurzen Beiträgen praxisorientierten Anleitungen und Anregungen einen großen Raum geben, dabei das jeweilige Thema jeweils von verschiedenen Seiten beleuchtend.

In dieser Ausgabe ist es uns wiederum gelungen, den Praxisschwerpunkt Palliativmedizin und Onkologie vielseitig zu bestücken, außerdem konnten wir dem Schwerpunkt erfreulicherweise eine Übersichtsarbeit aus dem Blickwinkel der japanischen Medizin zur Seite stellen, der weitere Möglichkeiten eines integrativen Ansatzes aufzeigt.

Was die Wissenschaft betrifft, werden wir weiterhin themenbezogen unter der Rubrik „Wissenschaft für die Kitteltasche“ den aktuellen Publikationsstand der Akupunktur zu bestimmten Fachgebieten aufbereiten, im „Journal Club“ Ihnen die Möglichkeit geben, unsere Beurteilung neuerer Studien zur Akupunktur zu lesen, und - last, not least - versuchen, Ihnen mehr Originalbeiträge und Übersichtsarbeiten anbieten zu können.

\section{Unser generelles Anliegen}

Akupunktur ist etabliert. Die Anwendung der Akupunktur ist zwischenzeitlich methodisch immer differenzierter geworden. Die Zahl der Publikationen steigt. Die Indikationen gehen sowohl im deutschen Sprachraum wie auch international weit über das hinaus, was im Rahmen der Modellversuche geforscht und für würdig befunden wurde, im Rahmen der gesetzlichen Krankenversicherung in der Bundesrepublik Deutschland als erstattungsfähig zu gelten.

Weder aus wissenschaftlicher Sicht noch aus der Sicht ärztlicher Anwendung kann dabei die Erstattungsfähigkeit einer Akupunkturleistung im Rahmen des SGB V noch unsere Richtschnur sein. Das kürzlich ergangene Urteil des Bundessozialgerichts, in dem die Durchführung der Auflagen der Qualitätssicherungsvereinbarung Akupunktur konkretisiert werden, erscheint vor diesem Hintergrund ungeeignet, die Qualität der Akupunktur zu sichern. In der Urteilsbegründung vertritt der Senat die Auffassung, dass genau das, worauf wir in der medizinischen Ausbildung und ärztlichen Tätigkeit höchsten Wert legen, nämlich die sorgfältige Anamnese und das dokumentierte Patientengespräch, allein nicht geeignet sei, in diesem Kontext eine Akupunkturanwendung zu begründen. Offensichtlich besteht im Zeitalter der Fake News von genannter Seite ein Misstrauensvorschuss, der interessengeleitete Dokumentation unterstellt. Man stelle sich vor, die Anforderung einer Gegenprüfung und flankierenden weiteren Dokumentation würde für alle medizinischen Handlungsfelder gelten.

Die Akupunktur ist ein vielfältiges therapeutisches Instrument, kein Fachgebiet, bestenfalls ein Querschnittsfach. Ich wünsche mir, dass wir die Basis wissenschaftlicher Daten und empirischer Erkenntnisse zur Akupunktur und zu verwandten Verfahren verbreitern. Hierzu möchte ich Kolleginnen und Kollegen ermuntern, die Kongresse und Fortbildungen in ihren jeweiligen Fachgebieten auf unsere Fragen hin zu überprüfen, so zum Beispiel: Inwieweit werden relevante Erkenntnisse für die Akupunktur präsentiert? Wie stellen sich therapeutische Neuerungen und ihre Wirksamkeit im Vergleich mit den vorliegenden Erkenntnissen zur Akupunkturanwendung dar?

Ich sehe meine Aufgabe als Chefredakteur darin, alle Leser und Leserinnen sowie natürlich auch die Redaktion in dieser Richtung zu unterstützen. In diesem Sinne hoffe ich von allen Seiten auf Ihre rege Mitwirkung am Gelingen dieser Zeitschrift!

Ihr

Jürgen Bachmann

Interessenkonflikt. J. Bachmann gibt an, dass kein Interessenkonflikt besteht. 